\title{
Sexual Differences in Physiological and Transcriptional Responses to Salinity Stress of Salix linearistipularis
}

\author{
Shuang Feng ${ }^{1}$, Hongwei Sun ${ }^{2}$, Hongping $\mathrm{Ma}^{2}$, Xin Zhang ${ }^{2}$, Shurong Ma1 ${ }^{1}$ Kun Qiao², \\ Aimin Zhou ${ }^{2}$, Yuanyuan Bu${ }^{1 *}$ and Shenkui Liü*
}

${ }^{1}$ Key Laboratory of Saline-Alkali Vegetation Ecology Restoration, Ministry of Education, Northeast Forestry University, Harbin, China, ${ }^{2}$ College of Horticulture and Landscape Architecture, Northeast Agricultural University, Harbin, China, ${ }^{3}$ The State Key Laboratory of Subtropical Silviculture, Zhejiang Agriculture and Forestry University, Hangzhou, China

\section{OPEN ACCESS}

Edited by:

Andrew Wood,

Southern Illinois University

Carbondale, United States

Reviewed by:

Sheng Zhang,

Institute of Mountain Hazards and Environment (CAS), China

Weiqiang $\mathrm{Li}$,

RIKEN, Japan

${ }^{*}$ Correspondence:

Yuanyuan Bu

yuanyuanbu@nefu.edu.cn

Shenkui Liu

shenkuiliu@nefu.edu.cn

Specialty section:

This article was submitted to

Plant Abiotic Stress,

a section of the journal

Frontiers in Plant Science

Received: 06 December 2019

Accepted: 29 September 2020

Published: 19 October 2020

Citation

Feng S, Sun $H$, Ma H, Zhang $X$, Ma S, Qiao K, Zhou A, Bu Y and Liu S

(2020) Sexual Differences

in Physiological and Transcriptional Responses to Salinity Stress of Salix

linearistipularis.

Front. Plant Sci. 11:517962. doi: 10.3389/fp/s.2020.517962
Willow (Salix), a dioecious plant, is an important ornamental tree species in the world. Salix linearistipularis, a perennial woody plant species naturally distributed on the Songnen Plain saline-alkali land in northeast China, has a high saline condition. To study the sexual differences of $S$. linearistipularis in salinity tolerance, the physiological and transcriptional responses to salinity were compared between female and male cuttings. Under salinity stress, the female leaves exhibited higher superoxide dismutase and peroxidase activities and photosynthetic capacity, and lower $\mathrm{H}_{2} \mathrm{O}_{2}$ contents than those of male leaves. Under salinity stress, sodium $\left(\mathrm{Na}^{+}\right)$accumulation in female leaves was lower than that in the male leaves. The non-invasive micro-test showed that the net $\mathrm{Na}^{+}$efflux in the salt-treated female roots was higher than that in male roots. Physiological responses revealed that female cuttings were more tolerant than males, which may be mainly due to females having lower leaf $\mathrm{Na}^{+}$accumulation and higher root $\mathrm{Na}^{+}$efflux capacity than males. Transcriptional analyses showed that 108 differentially expressed salt-responsive genes were identified in both female and male roots; most of these showed sexual differences in expression patterns under salinity stress. RNAseq combined with qPCR analysis showed that the salt-induced expression of four $\mathrm{Na}^{+} / \mathrm{H}^{+}$antiporter $(\mathrm{NHX})$ genes $(\mathrm{S} / \mathrm{NHX} 3,5,6,7)$ in female roots was higher than that in male roots. Transcriptional analyses revealed that the higher $\mathrm{Na}^{+}$efflux capacity in female roots than in male roots may be closely related to the differential expression of salt-responsive genes, especially $N H X$ genes.

Keywords: dioecious, Salix linearistipularis, salinity tolerance, sexual differences, salt-responsive genes

\section{INTRODUCTION}

Soil salinity is one of the major environmental factors influencing the productivity of agriculture and forestry. To survive under adverse conditions, plants have evolved intricate salt tolerance mechanisms, especially halophytes growing in high saline soils (Deinlein et al., 2014). Generally, halophytes can exclude more sodium $\left(\mathrm{Na}^{+}\right)$through their roots than glycophytes under salinity stress (Garthwaite et al., 2005). Investigation of salt tolerance mechanisms could facilitate the identification of plants that better cope with salinity. Moreover, the salt tolerant plants are an important resource for the exploitation and improvement of saline soils. 
Willow (Salix), as one of the most important landscaping greening tree species, has wide distribution and high adaptability (Zhang et al., 2017). Because of their rapid growth, high biomass yield, and ease of propagation, willows are important wood resources for both bioenergy production and afforestation (Greger and Landberg, 1999; Karp et al., 2011). Furthermore, willow species are extensively used in phytoremediation activities (Tozser et al., 2017). Salix linearistipularis (Franch.) Hao, a member of the Salicaceae family, is a woody plant naturally distributed in the Songnen Plain saline-alkali land in the northeast of China. S. linearistipularis exhibits high tolerance to salinity; therefore, it can be used for the greening and afforestation of saline-alkali land, which highlight its high and has great ecological and economic potential.

Salix linearistipularis is a dioecious plant. In many dioecious plant species, there are differences in morphology, physiology, and heredity, as well as environmental adaptability between female and male plants (Eckhart and Chapin, 1997; Li et al., 2007; Melnikova et al., 2017). For example, in poplars (Populus spp.) from the Salicaceae family, many studies have shown sexual differences between females and males in morphological, physiological, and transcriptional responses to environmental stress (Chen F. et al., 2010; Chen L. et al., 2010; Zhang et al., 2011; Jiang et al., 2012; Peng et al., 2012; Melnikova et al., 2017; Song et al., 2019). In P. cathayana, the growth rate, the leaf morphology changes, the dry matter and the $\mathrm{Na}^{+}$accumulation, and the photosynthetic capacity in female and male cuttings under salinity stress were significantly different (Chen F. et al., 2010). Furthermore, the difference in degradation rate and abundance of proteins involved in photosynthesis, hydrogen peroxide $\left(\mathrm{H}_{2} \mathrm{O}_{2}\right)$ scavenging, and stress response between female and male cuttings was observed under salinity stress (Chen et al., 2011). In P. yunnanensis, female cuttings exhibited higher sensitivity to salinity and drought than male cuttings, as well as higher sensitivity to the combination of salinity and elevated $\mathrm{CO}_{2}$ levels (Chen L. et al., 2010; Li et al., 2013). The transcription profiling revealed a difference in the expression of most genes involved in photosynthesis in both female and male $P$. yunnanensis under salinity stress (Jiang et al., 2012). Moreover, male P. deltoides cuttings exhibited competitive advantages over females under salinity stress (Li et al., 2016). Overall, these studies have shown the sexual difference in salinity tolerance between female and male plants in the genus Populus. The genus Populus and the genus Salix are members of Salicaceae. Unlike the male-biased Populus, Salix shows a female-biased sex ratio in its natural habitats (Dudley, 2006; Ueno et al., 2007; Hughes et al., 2010; Liao et al., 2019). A recent study has demonstrated that Salix paraplesia females exhibit superior drought tolerance and self-protective capacity to males at high altitudes (Liao et al., 2019). However, it remains unclear whether there are sexual differences in salinity tolerance between females and males in the genus Salix.

To investigate potential differences in physiological and transcriptional response of female and male $S$. linearistipularis cuttings, we measured some physiological parameters (including antioxidant enzyme activity, chlorophyll pigments, $\mathrm{H}_{2} \mathrm{O}_{2}$, and sodium $\left(\mathrm{Na}^{+}\right)$contents, and root $\mathrm{Na}^{+}$efflux rate) of female and male cuttings following exposure to salinity stress. In addition, we analyzed the transcription and expression profiles of salt-responsive genes in female and male roots exposed to salinity stress. The results of the present study could enhance our understanding of sexual differences in the genus Salix and facilitate its effective exploitation.

\section{MATERIALS AND METHODS}

\section{Plant Materials and Growth Conditions}

Annual female and male cuttings from S. linearistipularis trees (sampled in five populations) were collected from Anda Experimental Base of Northeast Forestry University of China (Anda City, Heilongjiang Province; $46^{\circ} 27, \mathrm{~N}, 125^{\circ} 22$, E). The cuttings were grown in a greenhouse under a 12-h light/12-h dark photoperiod ( $100 \mu \mathrm{mol} \mathrm{m}{ }^{-2} \mathrm{~s}^{-1}$ light) at $24^{\circ} \mathrm{C}$. Female and male cuttings were planted in plastic pots containing the same homogenized soil. After 1 month, healthy plants with equivalent height and similar crown size were selected for the experiments. A female tree and a male tree from each population were also randomly selected for different treatments.

\section{Measurement of Antioxidant Enzyme Activities and $\mathrm{H}_{2} \mathrm{O}_{2}$ and Malondialdehyde Contents}

The leaves of female and male plants were used to prepare leaf disks $\left(1 \mathrm{~cm}^{2}\right)$, which were immediately immersed in different concentrations of $\mathrm{NaCl}$ solution $(0,50,100,200$, 300 , and $400 \mathrm{mM}$ ) for $48 \mathrm{~h}$. Water was used as the control. A total of $100 \mathrm{mg}$ of the salt-treated and control samples were collected and weighed. The superoxide dismutase (SOD) and peroxidase (POD) activities, and $\mathrm{H}_{2} \mathrm{O}_{2}$ and MDA contents were measured using detection kits (SOD-1-Y, POD-1-Y, $\mathrm{H}_{2} \mathrm{O}_{2}-1$ Y, and MDA-1-Y; Comin, Suzhou, China), according to the manufacturer's instructions.

\section{Measurement of Chlorophyll Content and Chl Fluorescence Parameters}

Chlorophyll (Chl) was extracted from leaf samples using $80 \%$ ice-cold acetone. The absorbances of $\mathrm{Chl} \mathrm{a}(646 \mathrm{~nm})$ and $\mathrm{Chl} b$ $(663 \mathrm{~nm})$ were determined using a UV/Vis spectrophotometer. The total Chl content was the sum of $\mathrm{Chl}$ a and $\mathrm{Chl}$ b. The maximal photochemical efficiency $\left(F_{v} / F_{m}\right)$, minimal fluorescence yield $\left(F_{0}\right)$, and maximal fluorescence yield $\left(F_{m}\right)$ were measured using an Imaging-PAM Chlorophyll Fluorometer (Walz, Germany) as described previously (Wang et al., 2014).

\section{Measurement of $\mathrm{Na}^{+}$Contents}

Female and male cuttings were exposed to 0 and $100 \mathrm{mM} \mathrm{NaCl}$ salinity solution for 12 and $48 \mathrm{~h}$. After salinity treatment, the leaf samples of the cuttings were collected and dried. The dried samples were weighed and then digested in $8 \mathrm{~mL} \mathrm{HNO}_{3}$ and $3 \mathrm{~mL} \mathrm{H}_{2} \mathrm{O}_{2}$ for $50 \mathrm{~min}$ at $180^{\circ} \mathrm{C}$ using a microwave digestion instrument (Milestone, Italy). The $\mathrm{Na}^{+}$contents in the leaves were measured by inductively coupled plasma optical emission spectrometry (ICP-OES, Perkin Elmer, United States). 


\section{Net $\mathrm{Na}^{+}$Flux Measurements}

Net fluxes of $\mathrm{Na}^{+}$were measured using Non-Invasive Microtest Technology (NMT) (NMT100 Series, YoungerUSA LLC, Amherst, MA, United States), as described previously (Sun et al., 2009; Zhou et al., 2018). Hydroponic female and male cuttings were exposed to 0 or $100 \mathrm{mM} \mathrm{Na}^{+}$for $12 \mathrm{~h}$, and root segments were immobilized in the measuring solution $(0.1 \mathrm{mM} \mathrm{KCl}$, $0.1 \mathrm{mM} \mathrm{CaCl}_{2}, 0.1 \mathrm{mM} \mathrm{MgCl}_{2}, 0.5 \mathrm{mM} \mathrm{NaCl}$, and $0.3 \mathrm{mM} \mathrm{MES}$, $\mathrm{pH}$ 5.8) to measure the $\mathrm{Na}^{+}$flux. Each sample was measured continuously for $9 \mathrm{~min}$ and each root zone for $3 \mathrm{~min}$. The $\mathrm{Na}^{+}$ flux rate, based on the voltages monitored between two points ( 0 and $30 \mu \mathrm{m}$ ), was calculated using iFluxes/imfluxes v1.0 software (YoungerUSA LLC, Amherst, MA, United States). The calibration slope for $\mathrm{Na}^{+}$was $58.59 \mathrm{mV} /$ decade. Six biological repeats were performed for each analysis. Ion flux was calculated based on Fick's law of diffusion:

$$
J=-D(d c / d x)
$$

where $J$ represents the ion flux in the $x$ direction, $d c / d x$ is the ion concentration gradient, and $D$ is the ion diffusion constant in a particular medium.

\section{RNA-Seq and Transcriptome Data Processing}

Hydroponic cuttings of male and female plants were exposed to 0 or $100 \mathrm{mM} \mathrm{NaCl}$ for 6,12 , and $24 \mathrm{~h}$. Three independent experiments were performed for each treatment condition. Root samples from all cuttings were cut and immediately stored at $-80^{\circ} \mathrm{C}$ until required for RNA extraction. Total RNA from the root samples was extracted using TRIzol reagent (Invitrogen, Carlsbad, CA, United States). The quality and integrity of the RNA samples was confirmed and assessed, no sign of degradation was observed. cDNA library construction and Illumina sequencing were performed according to the manufacturer's instructions (Illumina, San Diego, CA, United States). RNA sequencing (RNA-seq) was performed by the Beijing Genomic Institute (BGI, Shenzhen, China).

The de novo assembly of RNA-seq in the absence of a reference genome was accomplished using Trinity (Grabherr et al., 2011). Trinity combines the reads with a certain length of overlap to form longer fragments, known as contigs. These contigs were subjected to sequence clustering to form longer sequences. Such sequences were defined as unigenes (Zhou et al., 2017). For unigenes annotation, seven public databases were used, including
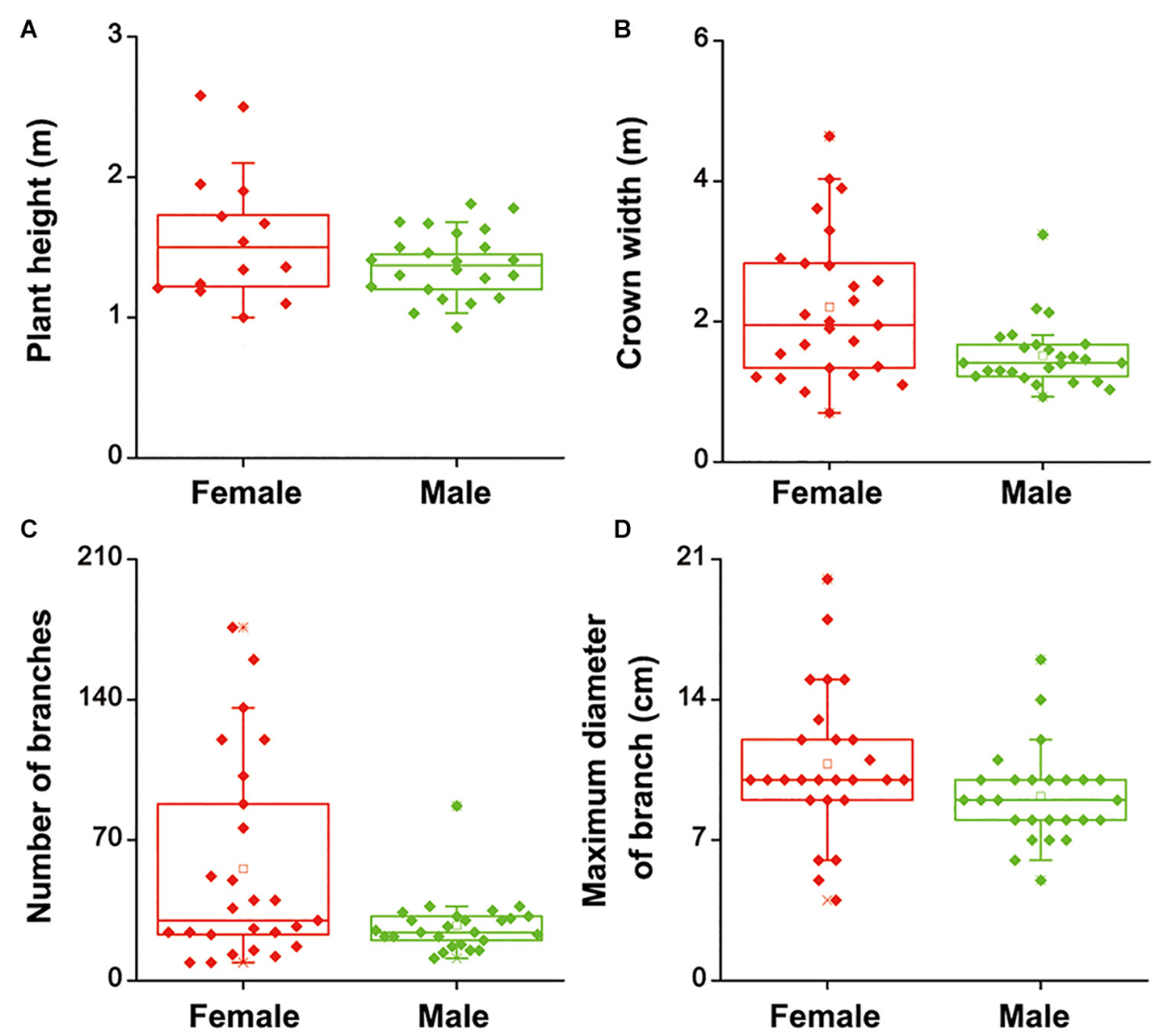

FIGURE 1 | Height (A), crown width (B), number of branches (C), and maximum diameter of branches (D) of female and male plants of S. linearistipularis from three natural populations distributed in saline-alkali soil. In the box plots, the hollow square () show the medians and center lines show the mean value; box limits indicate the 25th and 75th percentiles as determined by R software; whiskers extend 1.5 times the interquartile range from the 25th and 75 th percentiles, and individual data points are represented by solid square. 
the NCBI non-redundant protein sequence (NR), Swiss-Prot protein, euKaryotic Ortholog Groups (KOG), NCBI nucleotide sequence (NT), Kyoto Encyclopedia of Genes and Genomes (KEGG), protein families (Pfam), Gene Ontology (GO), and Intersection databases. The RNA-seq data were deposited in the NCBI Gene Expression Omnibus (GEO) with accession number GSE138551.

\section{Analysis of Differentially Expressed \\ Genes}

Transcriptome data processing and differentially expressed gene (DEG) analysis were performed as previously described (Zhou et al., 2017). The expression levels of the unigenes were calculated using the Reads per kilobase per Million reads (RPKM) method (Mortazavi et al., 2008). DEGs were screened with a false discovery rate (FDR) threshold of 0.05 or less and an absolute $\log 2$ ratio of 1 or more. All the DEGs were mapped to each term of the GO databases, and significant pathways were defined based on a corrected $P$-value $\leq 0.05$.

\section{qPCR Expression Analysis}

Expression levels of five sodium/hydrogen antiporter (NHX) genes (Unigene43275, CL3276, CL2217, CL7345, and CL9910) were investigated by qPCR. The SlActin (CL19537) was used as the internal reference gene. The relative gene expression levels
A

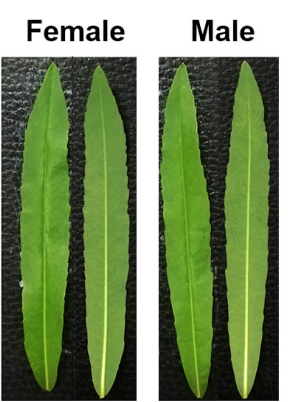

C

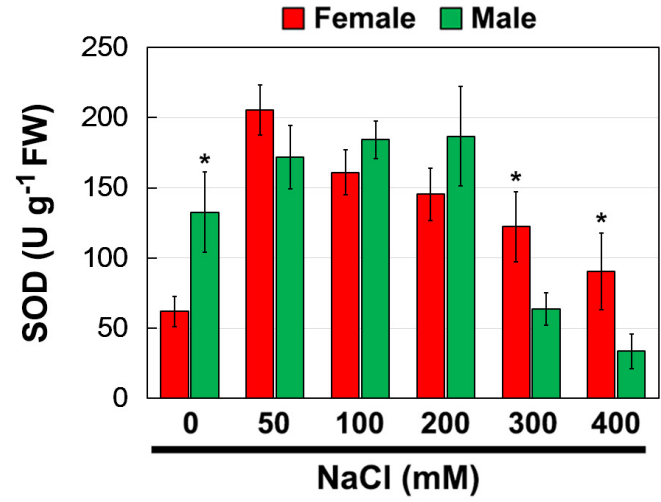

E

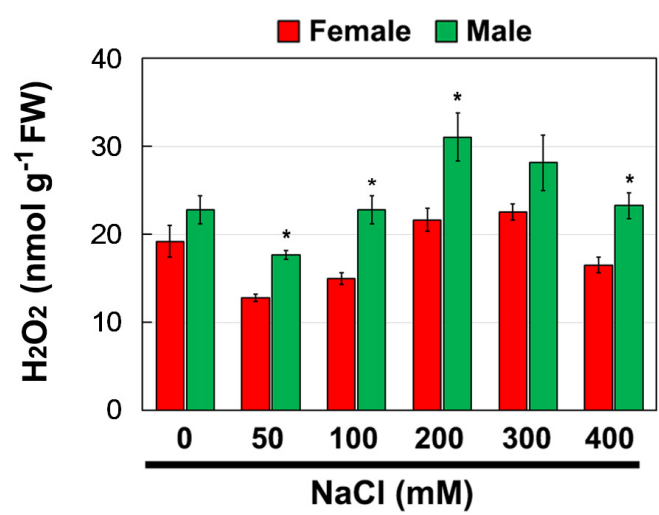

B

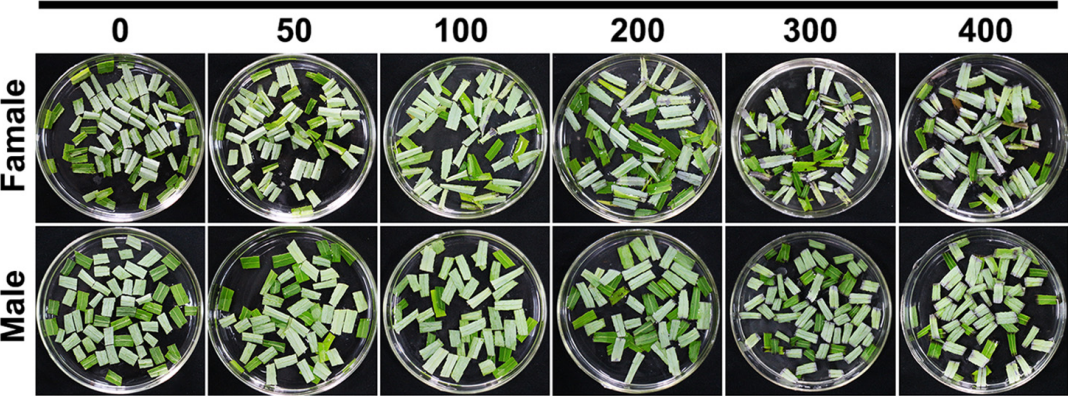

D

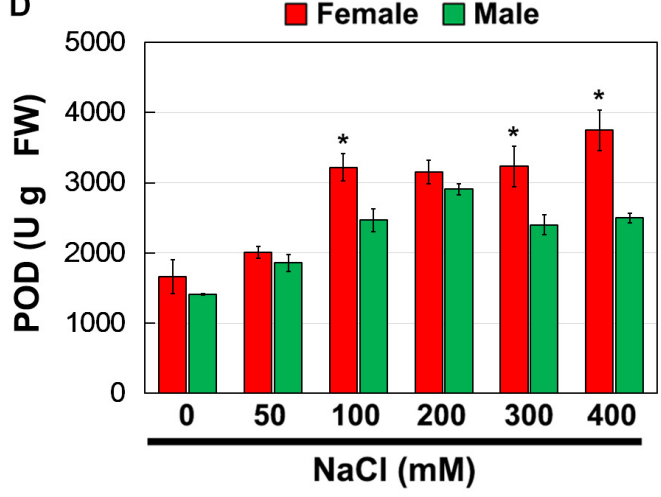

$\mathbf{F}$

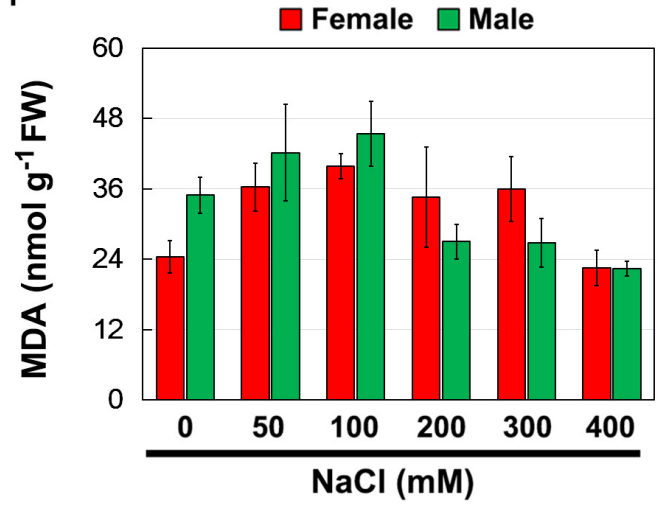

FIGURE 2 | Phenotype (A,B), SOD (C), and POD (D) activities, and contents of $\mathrm{H}_{2} \mathrm{O}_{2}$ (E) and MDA (F) in leaves of female and male plants of S. linearistipularis under control and salinity stress (50 to $400 \mathrm{mM} \mathrm{NaCl})$. Error bars represent $S E(n=5)$. Asterisks indicate significant differences between female and male plants $(* P<0.05 ;$ Student's $t$-test). Fw, fresh weight. 
were quantified using the delta-delta-Ct method. The qPCR primers used are listed in Supplementary Table S1.

\section{Statistical Analyses}

All experiments performed at least three biological and at least three technical repeats. Statistically significant differences were calculated based on the Student's $t$-test, with $P<0.05\left(^{*}\right)$ using one-way Analyses of Variance in SPSS (SPSS Inc., Chicago, IL, United States).

\section{RESULTS}

\section{Comparison Between Sexes in Leaf Physiological Traits}

To investigate whether there were differences in salinity tolerance between female and male of S. linearistipularis plants, growth phenotypes from five populations in saline-alkali habitats were first investigated and compared. The average plant height, crown width, branch number, and maximum branch diameter of female plants were higher than those of male plants (Figures 1AD). There were no significant morphological differences in female and male leaves (Figure 2A). The physiological traits of female and male leaves exposed to salinity $(\mathrm{NaCl})$ stress were measured. Under the control conditions $(0 \mathrm{mM})$, the male leaves showed significantly higher SOD activity than the female leaves (Figure 2B). After 50 to $200 \mathrm{mM} \mathrm{NaCl}$ treatment, there were no significant differences between sexes. At higher $\mathrm{NaCl}$ concentrations (300 and $400 \mathrm{mM}$ ), female leaves exhibited significantly higher SOD activity than males (Figure 2C). Under salinity stress, female leaves exhibited generally higher POD activity and lower $\mathrm{H}_{2} \mathrm{O}_{2}$ content than male leaves. However, there were no significant differences between female and male leaves under the control $(0 \mathrm{mM})$ condition (Figures 2D,E). In addition, there were no significant differences in leaf MDA contents between the females and males under both the control and salinity stress (Figure 2F).

Further, the total Chl content and $\mathrm{Chl}$ fluorescence parameters of female and male leaves exposed to salinity stress were measured (Figure 3A). Salinity stress decreased total Chl content in both female and male leaves, but there were no significant differences between them under the control and salinity stress treatments (Figure 3B). Under the control conditions, there was no significant difference in $F_{0}, F_{m}$, and $F_{v} / F_{m}$ between the female and male leaves. However, under salinity stress, the $F_{0}$ and $F_{m}$ in male leaves were higher than those in female leaves, while the $F_{v} / F_{m}$ in male leaves was significantly lower than that in female leaves (Figures 3C-E).

\section{Comparison Between Sexes in Leaf $\mathrm{Na}^{+}$ Accumulation and Root $\mathrm{Na}^{+}$Efflux}

When salinity $(100 \mathrm{mM} \mathrm{NaCl})$ was applied into the soil, the $\mathrm{Na}^{+}$accumulation in the female and male leaves increased. After 12 and $48 \mathrm{~h}$ of $\mathrm{NaCl}$ treatment, the $\mathrm{Na}^{+}$accumulation
A

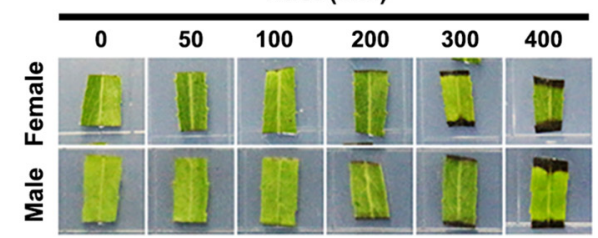

인

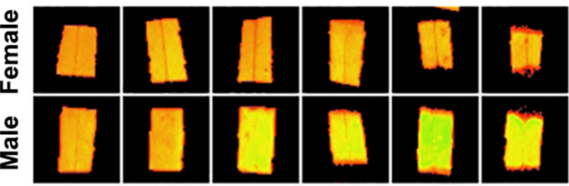

도|

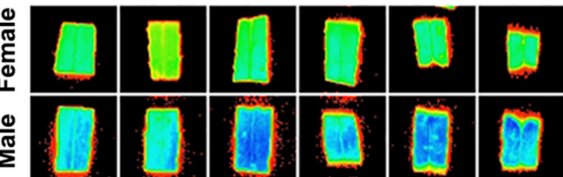

닌

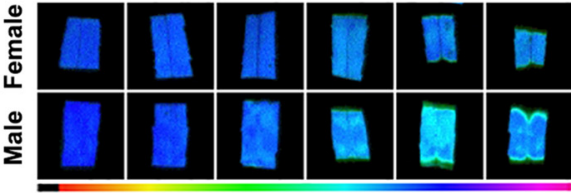

B

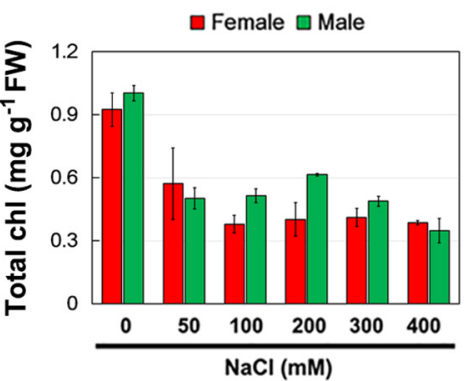

D

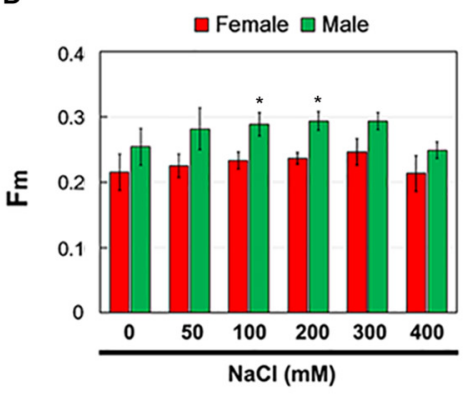

C

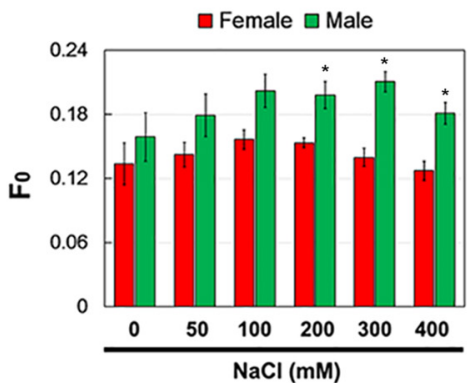

E

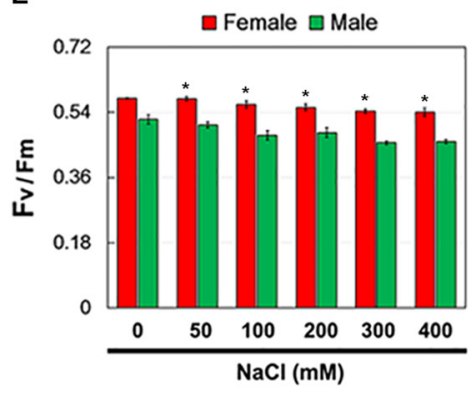

FIGURE 3 | Chlorophyll (Chl) contents and Chl fluorescence parameters in leaves of female and male plants of S. linearistipularis under control and salinity stress (50 to $400 \mathrm{mM} \mathrm{NaCl}$ ). (A) Minimum Chl fluorescence $\left(F_{0}\right)$, maximal Chl fluorescence ( $\left.F \mathrm{~m}\right)$, and maximal photochemical efficiency (Fv/Fm) images. The pseudocolored bar depicted at the bottom of the panel ranges from 0 (black) to 1.0 (purple). Chl content (B) and quantification of Chl fluorescence parameters $F_{0}$ (C), $F_{m}$ (D) and $F_{v} / F_{m}(\mathbf{E})$. Error bars represent $S E(n=6)$. Asterisks indicate significant differences between female and male plants $\left({ }^{*} P<0.05 ;\right.$ Student's $t$-test). FW, fresh weight. 
in male leaves was significantly higher than that in female leaves. However, there were no significant differences in $\mathrm{Na}^{+}$ accumulation between the female and male leaves under the untreated control conditions (Figure 4).

Using the NMT technique, the net $\mathrm{Na}^{+}$flux in female and male roots under salinity stress was monitored (Figure 5A). Transfer

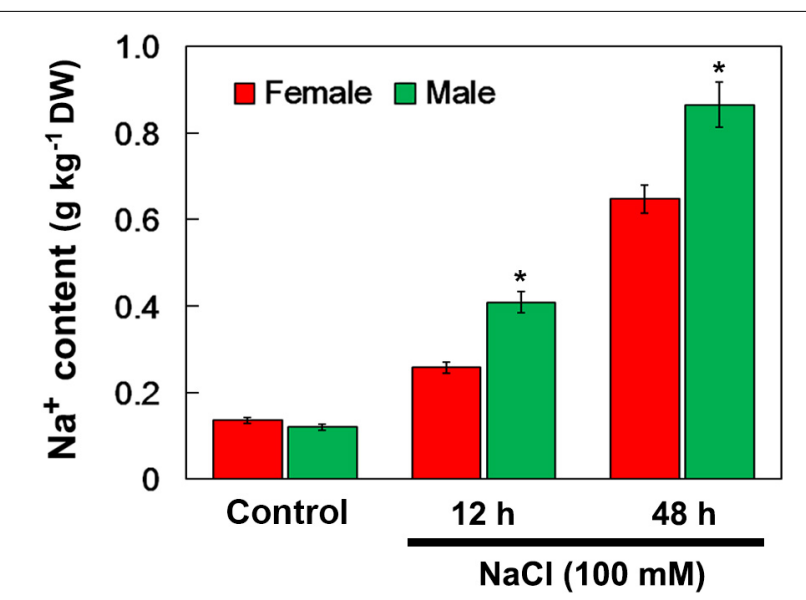

FIGURE 4 | $\mathrm{Na}^{+}$contents in leaves of female and male plants of S. linearistipularis under control and salinity stress (irrigation with $100 \mathrm{mM}$ $\mathrm{NaCl})$. Leaves were sampled 0 (control), 12 and $48 \mathrm{~h}$ after the initiation of salinity treatment. Error bars represent $S E(n=3)$. Asterisks indicate significant differences between female and male plants $\left({ }^{*} P<0.05\right.$; Student's $t$-test). DW, dry weight. of $\mathrm{NaCl}$-treated roots to $\mathrm{Na}^{+}$-free solution caused a significant $\mathrm{Na}^{+}$efflux in both the female and male roots, including the root meristem zone (Mez), elongation zone (Elz), and mature zone (Maz) (Figure 5B). The rate of net $\mathrm{Na}^{+}$efflux in female roots was significantly higher than that in male roots (Figure 5C). Under the control treatment, the rate of net $\mathrm{Na}^{+}$efflux in female and male roots was not significantly different (Figures 5D,E).

\section{Comparison Between Sexes in Transcriptional Profiling in Roots}

To investigate potential sexual differences in root $\mathrm{Na}^{+}$flux, transcriptional sequencing of female and male roots under control (CK) and salinity stress was performed. In total, 220,256 transcripts ( $85.56 \%$ of all transcripts) in the control and $\mathrm{NaCl}-$ treated roots of both female and male plants were annotated in the following public databases, including the NR, NT, Swiss-Prot, KEGG, KOG, Pfam, and GO (Table 1).

After merging transcripts with the same annotation, a total of 46,664 unigenes were obtained. Out of all the genes, 4,824 were identified in female plants, and 2,864 were identified in male plants, and 38,976 (83.5\% of all unigenes) were common to both the sexes (Figure 6A). Principal component analysis based on gene expression showed that the sequenced samples clustered well (Supplementary Figure S1). Using the RPKM method, the DEGs in female and male roots were screened. After 6, 12, and $24 \mathrm{~h}$ of $\mathrm{NaCl}$ treatment, 8,161 and 12,861 common DEGs were identified in female and male roots, respectively (Figure 6B). The GO enrichment analysis for DEGs showed that the response to the salt stress pathway was significantly enriched in both female
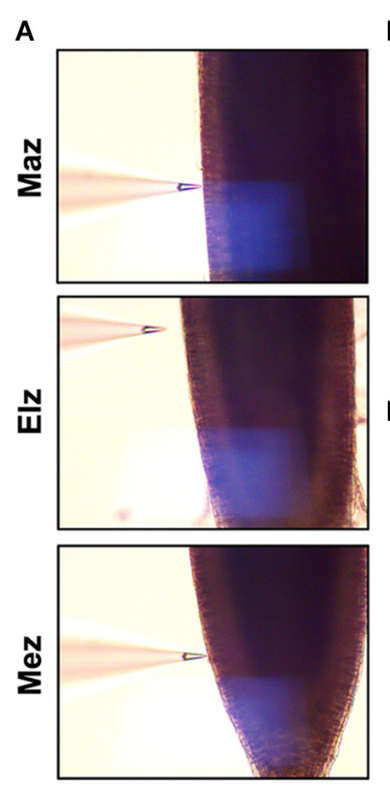

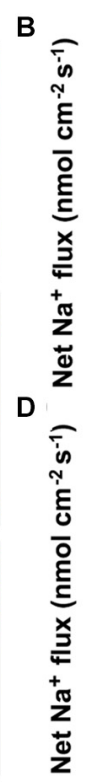

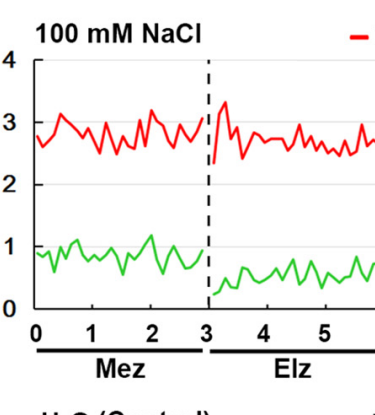
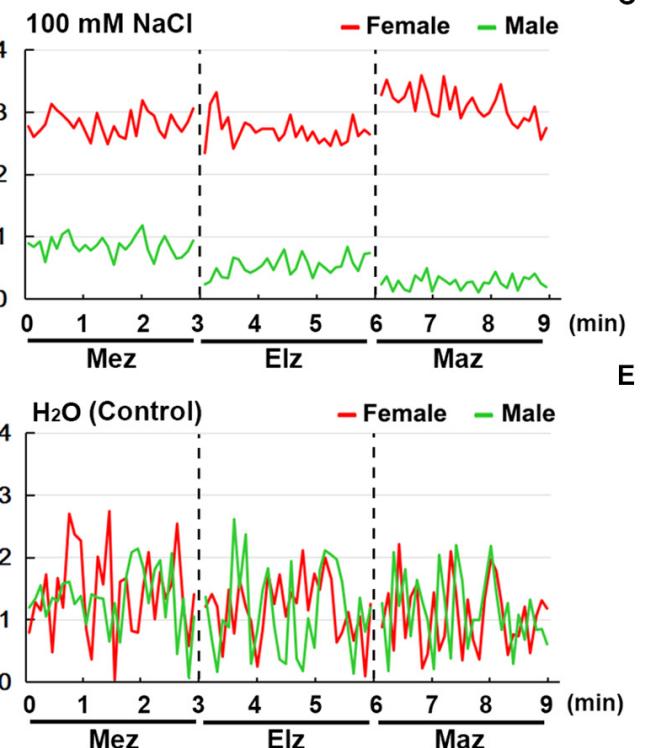

$c$

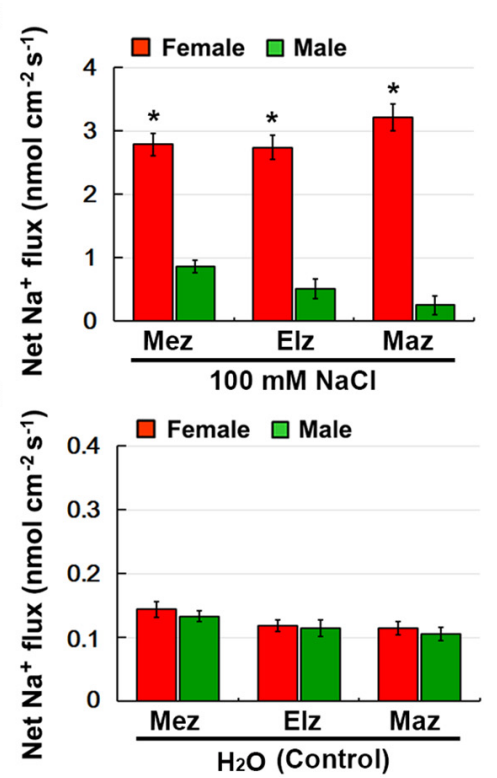

FIGURE 5 | Net fluxes of $\mathrm{Na}^{+}$in roots of female and male plants of $\mathrm{S}$. linearistipularis under control and salinity stress (100 mM NaCl for 12 h). (A) The root zones measured by NMT technology. Mez, Meristem zone; Elz, elongation zone; Maz, mature zone. (B) Net $\mathrm{Na}^{+}$fluxes in roots of female and male plants pretreated with $12 \mathrm{~h}$ incubation in $100 \mathrm{mM} \mathrm{NaCl}$. Continuous flux was recorded for $9 \mathrm{~min}$, 3 min per root zone. (D) The normal test solution (0.1 mM NaCl) was used as the control. $(\mathbf{C}, \mathbf{E})$ Mean $\mathrm{Na}^{+}$fluxes from the roots of female and male plants under the condition of $\mathrm{NaCl}(100 \mathrm{mM})$ treatment and control $(0.1 \mathrm{mM} \mathrm{NaCl})$. Error bars indicate SE $(n=6)$. Asterisks indicate significant differences between female and male plants ( ${ }^{*} P<0.05$; Student's $t$-test). 
TABLE 1 | Number of functional annotations for all of the transcripts in public databases.

\begin{tabular}{|c|c|c|c|c|c|c|c|c|}
\hline Annotated Database & NR & NT & SwissProt & KEGG & KOG & Pfam & GO & Overall \\
\hline Number of transcripts & 206,706 & 166,419 & 152,229 & 160,838 & 164,661 & 156,833 & 152,635 & 220,256 \\
\hline Percentage (\%) & $80.30 \%$ & $64.65 \%$ & $59.14 \%$ & $62.48 \%$ & $63.97 \%$ & $60.92 \%$ & $59.29 \%$ & $85.56 \%$ \\
\hline
\end{tabular}

A

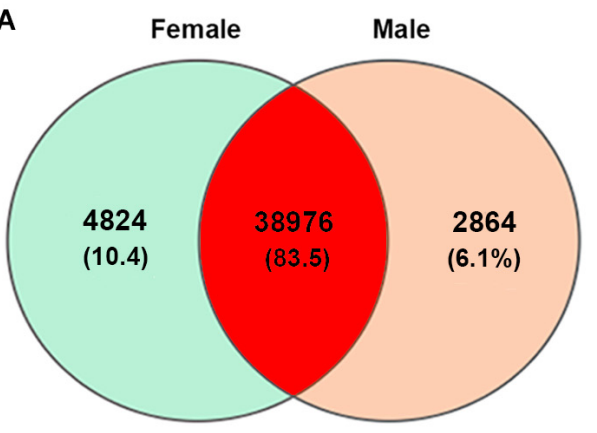

D

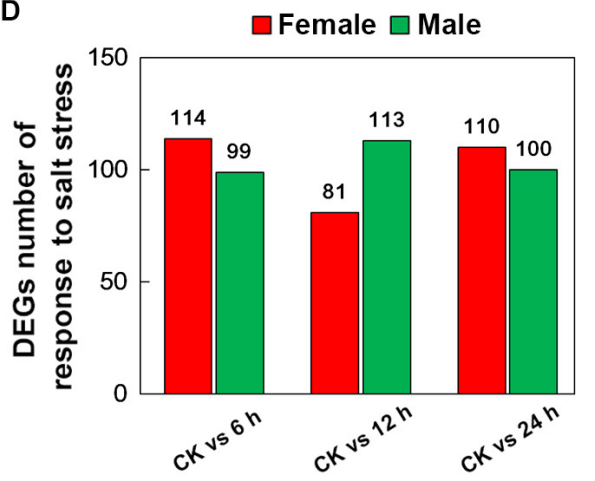

B

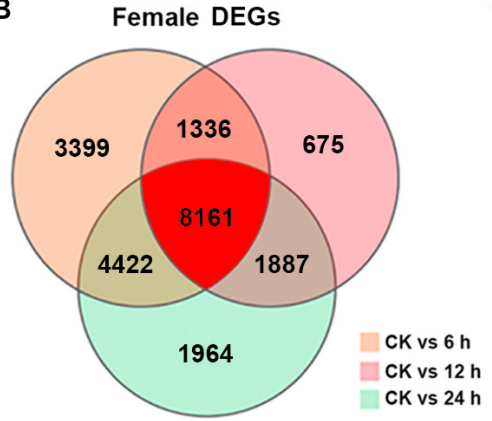

E

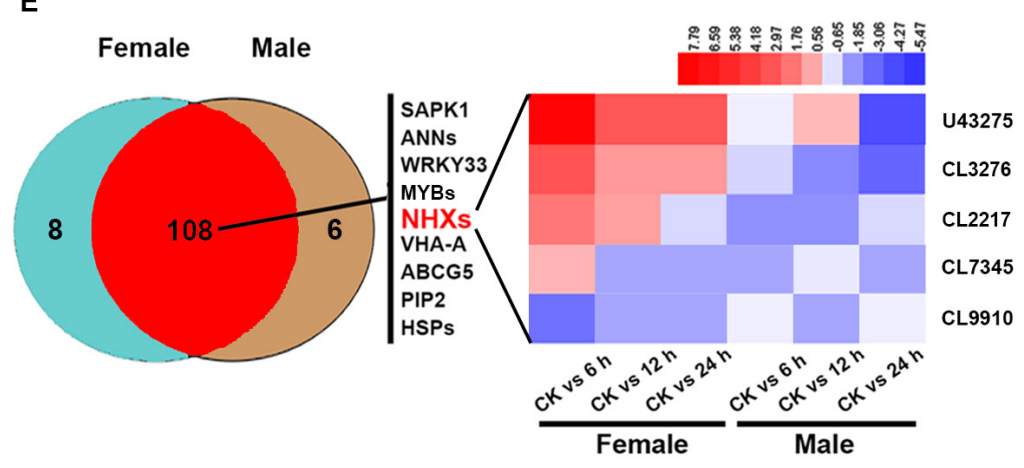

C

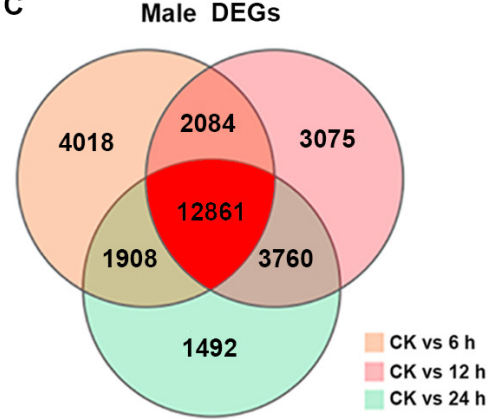

43275

CL3276

CL2217

7345

9910

FIGURE 6 | RNA-seq and analysis of salt-responsive genes in the roots of female and male plants of S. linearistipularis. (A) Venn diagrams of the total mapped genes expressed in the roots of female and male plants under control and salinity stress (100 mM NaCl). (B) Venn diagrams of DEGs identified in the roots of female and male plants under salinity stress for 6,12 , and $24 \mathrm{~h}$. We used a false discovery rate $\leq 0.01$ and the absolute value of log2Ratio $\geq 1$ as the threshold to judge the significance of the differences in gene expression. (C) Number of DEGs in response to salt stress in the roots of female and male plants. (D) Venn diagrams of genes responding to salt stress in the roots of male and female plants and expression profile of key salt-responsive genes (sodium/hydrogen antiporter, NHX). Red rectangles represent the up-regulation of genes, while blue rectangles represent down-regulation. CK, control.

and male plants (Supplementary Figure S2). At different time points of $\mathrm{NaCl}$ treatment, the number of DEGs involved in response to salt stress pathway was different between female and male plants (Figure 6C and Table 2). Furthermore, the number and expression levels of DEGs involved in reactive oxygen species pathways were different between the female and male plants (Table 2 and Supplementary Table S2). However, most of the DEGs involved in response to salt stress pathway were the same in both sexes; 108 DEGs are common to both sexes, 8 DEGs are specific to female plants, and 6 DEGs are specific to male plants (Figure 6D). The expression patterns of the common DEGs were different between the females and males under salinity stress (Supplementary Figure S3 and Supplementary Table S3). Notably, 5 NHX genes (U43275, CL3276, CL2217, CL7345, CL9910) were identified among the common DEGs, four of which (U43275, CL3276, CL2217, CL7345) exhibited higher expression levels in female roots than in male roots under salinity stress (Figure 6D). Sequence alignment showed that the five $N H X$ genes were highly homologous to PtNHX2, PtNHX3, PtNHX5,
PtNHX6, and PtNHX7/PtSOS1 of P. trichocarpa (Figure 7A). The qPCR results were generally in consistent with the RNA-seq data (Figures 7B-F).

\section{DISCUSSION}

\section{Sexual Difference in Physiological Response to Salinity Stress}

Salinity stress can cause ion toxicity and osmotic imbalances, leading to secondary oxidative stress in plants (Chaves et al., 2009). Thus, salt exclusion or reducing salt uptake, preventing oxidative damage are the key defense mechanisms for plant adaptation to salinity (Chaves et al., 2009). In our study, salinity treatment increased the $\mathrm{H}_{2} \mathrm{O}_{2}$ content and activated the activities of antioxidant enzymes, SOD and POD, in female and male leaves. However, the female leaves showed higher SOD and POD activity and lower $\mathrm{H}_{2} \mathrm{O}_{2}$ content than the male leaves under salinity stress (Figure 2). The results 


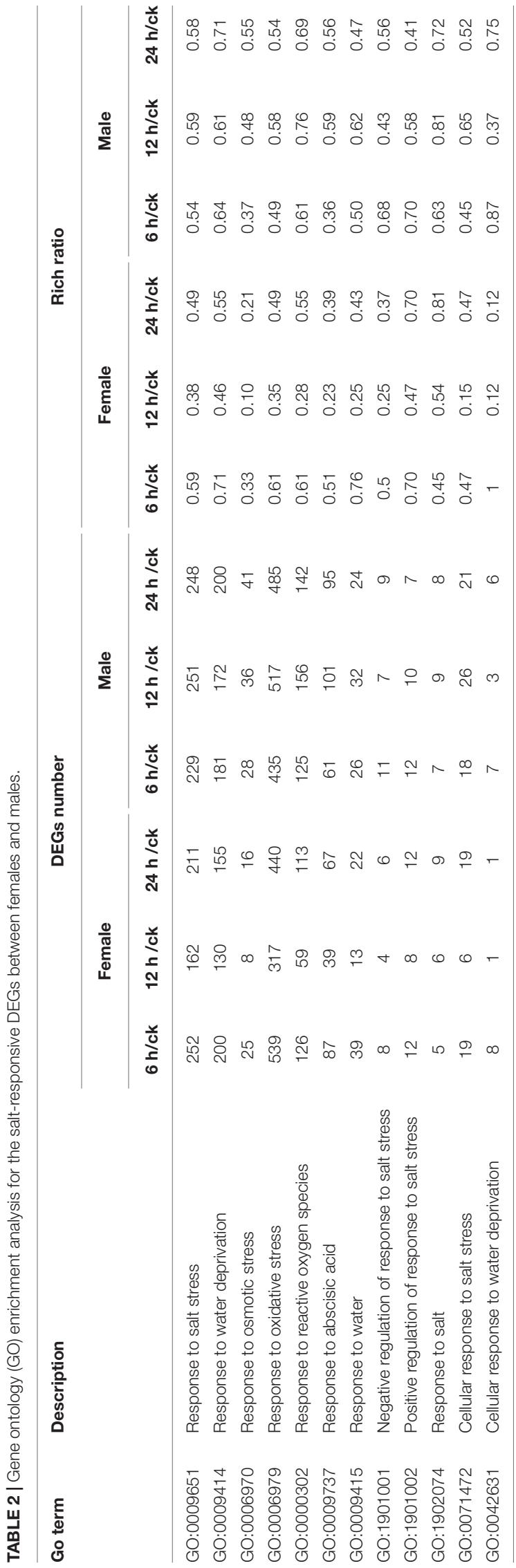

showed that the antioxidant response of female and male leaves was different, and the antioxidant capacity of female leaves was stronger than that of male leaves under salinity stress. Stress can cause the decrease in $F_{v} / F_{m}$, which can reflect the degree of PSII damage, and then be used as an indicator of the ability of plants to tolerate environmental stress (Maxwell and Johnson, 2000). Under salinity stress, $F_{v} / F_{m}$ in female leaves decreased more slowly than that in male leaves (Figure 3E). This result suggests that the damage of salinity to PSII in female leaves was weaker than that in male leaves.

\section{Sexual Difference in $\mathrm{Na}^{+}$Accumulation}

Long-term exposure of plants to salinity stress results in $\mathrm{Na}^{+}$ accumulation in leaves, leading to toxic symptoms (Munns and Tester, 2008; Rahnama et al., 2010). The $\mathrm{Na}^{+}$content in both female and male leaves significantly increased when exposed to salinity stress (Figure 4). However, under the untreated control conditions, there was no difference in $\mathrm{Na}^{+}$content between female and male leaves. The $\mathrm{Na}^{+}$accumulation in leaves is closely related to the $\mathrm{Na}^{+}$uptake rates by roots and the rate of $\mathrm{Na}^{+}$transport to shoots (Shi et al., 2002). NMT monitoring showed that the female roots had higher $\mathrm{Na}^{+}$efflux rate than that of the male roots under salinity stress (Figures 5B,C). These results showed that the $\mathrm{Na}^{+}$accumulation in leaves and $\mathrm{Na}^{+}$ efflux rate in roots were different in female and male plants, and less $\mathrm{Na}^{+}$accumulation in female leaves than male leaves may be related to higher $\mathrm{Na}^{+}$efflux rate in its roots under salinity stress.

\section{Sexual Difference in Transcriptional Response to Salinity Stress}

Transcriptional analysis showed that salinity induced the differential expression of a large number of genes in female and male roots (Figure 6B). At different time points after salt treatment, the number of salt response-related DEGs in female and male plants was different (Figure 6C). However, most of these salt response-related DEGs are common to both sexes, including SAPK1, WRKY33, VHA-A, ABCG5, and PIP2 genes, and many members from the $A N N, M Y B$, NHX, and HSP gene families (Figure 6D). These genes are reported to be involved in plant salt stress responses (Jiang and Deyholos, 2009; Matsuda et al., 2014; Zhu et al., 2015; Lv et al., 2017; Lou et al., 2018; Li et al., 2019). Under salt stress, $\mathrm{Na}^{+}$transport was directly regulated by the NHX family at endomembranes (Bassil and Blumwald, 2014). Genome analysis of multiple species has shown that the NHX family consists of about 5 to 9 members (Bassil and Blumwald, 2014). In P. trichocarpa, the NHX family consists of 8 members (Tian et al., 2017). Five NHX genes were identified from the transcriptome of S. linearistipularis roots by sequence alignment, and all of them appeared in common salt response-related DEGs of both sexes (Figure 6D). By phylogenetic analysis, the five SlNHXs were homologous to PtNHX2, PtNHX3, PtNHX5, PtNHX6, and PtNHX7/PtSOS1, respectively (Figure 7A). RNA-seq and qPCR experiments 
A

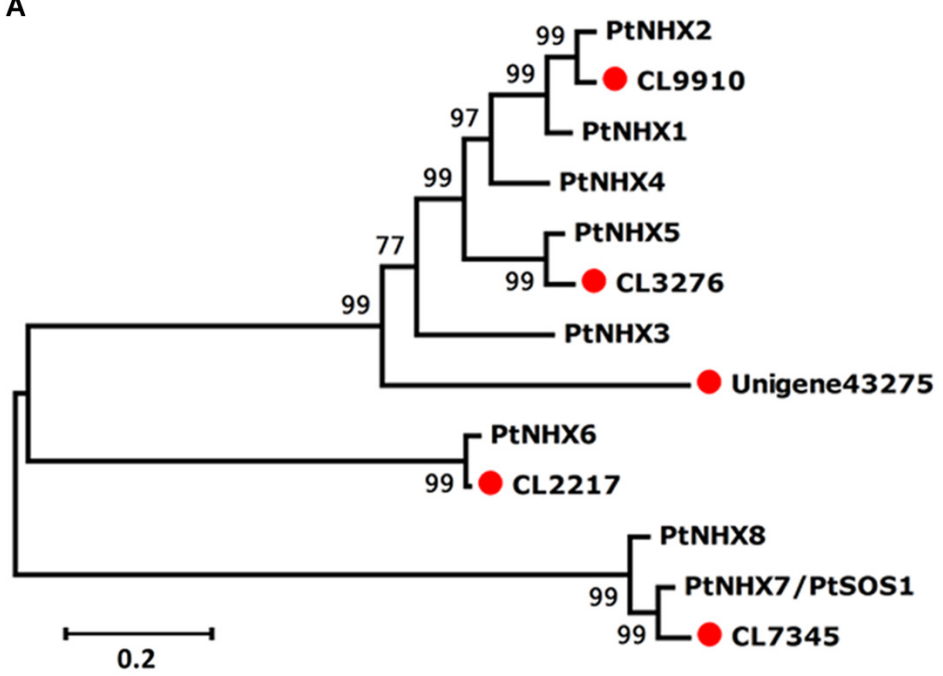

D

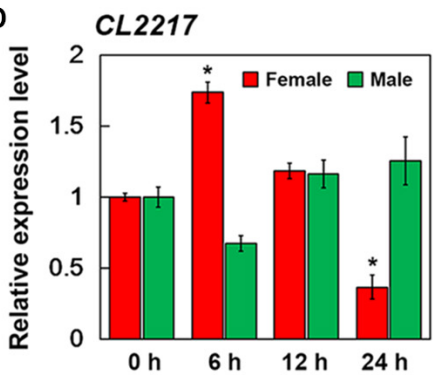

E

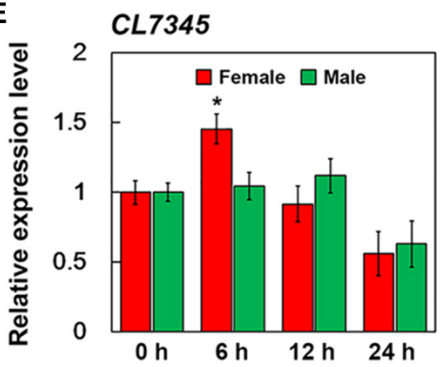

B
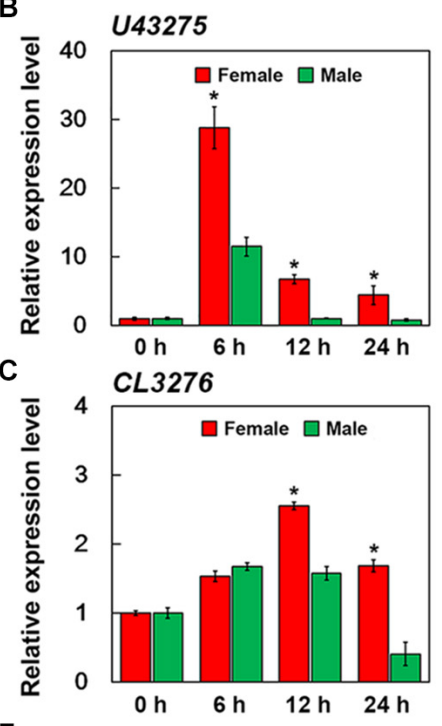

$\mathbf{F}$

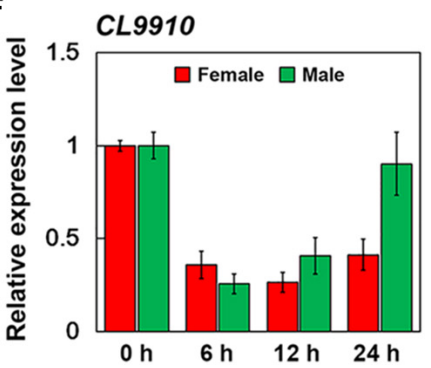

FIGURE 7 | qPCR verification of expression of five sodium hydrogen antiporter (NHX) genes in the roots of female and male plants of $S$. linearistipularis. (A) Phylogenetic trees of $S$. linearistipularis NHX proteins with P. trichocarpa NHX family. (B-F) Expression of five NHX genes in female and male roots under salinity stress for 6,12 , and $24 \mathrm{~h}$. Error bars indicate $S E(n=3)$. Asterisks indicate significant differences between female and male plants $\left({ }^{*} P<0.05\right.$; Student's $t$-test).

showed that the salt-induced expression of four NHX genes in female roots was higher than that in male roots (Figures 7BF). Thus, we speculated that the sexual difference of $\mathrm{Na}^{+}$ efflux between female and male roots may be closely related to the differential expression of NHXs in both sexes under salinity stress. These results suggest that the salinity tolerance mechanisms in female and male plants are the same, but the sexual differences in salinity tolerance between them may be caused by the differential expression of these saltresponsive DEGs, such as NHXs. However, the reasons for the differential expression of these salt-responsive DEGs in both sexes are unclear and need to be further investigated. Of course, we cannot completely rule out the regulatory roles of female and male specific DEGs in the sexual difference of salinity tolerance.

In $P$. cathayana, previous studies have shown that salinity tolerance in females was weaker than that in males (Chen F. et al., 2010). The result is different from our observation, suggesting that the salinity tolerance mechanism of females and males in $P$. cathayana and S. linearistipularis is different. This difference may be related to species characteristics and habitats. The leaf morphology of $P$. cathayana was significantly different between females and males, but there was no difference in the leaf morphology between females and males in S. linearistipularis. In addition, the habitats of $P$. cathayana and S. linearistipularis are different. Saline-alkali habitat may have led to the evolution of a salinity tolerance mechanism in S. linearistipularis, which is different from that in P. cathayana. This observation needs to be verified in more species from the genus Salix. Recent studies have shown that $S$. paraplesia females are more drought tolerant and better self-protective ability than males from high altitude (Liao et al., 2019).

\section{CONCLUSION}

We identified differences in salinity tolerance between female and male plants of $S$. linearistipularis by comparing the physiological traits and transcriptional profiling. In female roots, the differential expression of salt-response genes, especially $N H X$ s, may cause stronger $\mathrm{Na}^{+}$efflux, which further leads to less $\mathrm{Na}^{+}$accumulation in leaves than in male roots. Lower $\mathrm{Na}^{+}$accumulation in female leaves may be associated with higher antioxidant enzyme activity and lower $\mathrm{H}_{2} \mathrm{O}_{2}$ levels. It is inferred from the above results that female plants have stronger salinity tolerance than male plants. Therefore, in the 
afforestation process of $S$. linearistipularis for greening and ecological restoration of saline-alkali soil in the future, different salinity tolerance characteristics of female and male plants should be considered.

\section{DATA AVAILABILITY STATEMENT}

The datasets generated for this study can be found in the NCBI Gene Expression Omnibus (GEO), GSE138551.

\section{AUTHOR CONTRIBUTIONS}

SF, AZ, YB, and SL designed the experiments. SF, AZ, HS, $\mathrm{HM}$, and $\mathrm{XZ}$ performed the experiments. SF, SM, KQ, and YB analyzed the data. SF and AZ wrote the manuscript. All authors contributed to the article and approved the submitted version.

\section{FUNDING}

This work was supported by the Zhejiang Science and Technology Major Program on Agricultural New Variety Breeding of China (No. 2016C02056-1), the State Key Laboratory of Subtropical Silviculture Open Fund of China (No. KF201707),

\section{REFERENCES}

Bassil, E., and Blumwald, E. (2014). The ins and outs of intracellular ion homeostasis: NHX-type cation/H(+) transporters. Curr. Opin. Plant Biol. 22, 1-6. doi: 10.1016/j.pbi.2014.08.002

Chaves, M. M., Flexas, J., and Pinheiro, C. (2009). Photosynthesis under drought and salt stress: regulation mechanisms from whole plant to cell. Ann. Bot. 103, 551-560. doi: 10.1093/aob/mcn 125

Chen, F., Chen, L., Zhao, H., Korpelainen, H., and Li, C. (2010). Sex-specific responses and tolerances of Populus cathayana to salinity. Physiol. Plant 140, 163-173. doi: 10.1111/j.1399-3054.2010.01393.x

Chen, F., Zhang, S., Jiang, H., Ma, W., Korpelainen, H., and Li, C. (2011). Comparative proteomics analysis of salt response reveals sex-related photosynthetic inhibition by salinity in Populus cathayana cuttings. J. Proteome Res. 10, 3944-3958. doi: 10.1021/pr200535r

Chen, L., Zhang, S., Zhao, H., Korpelainen, H., and Li, C. (2010). Sexrelated adaptive responses to interaction of drought and salinity in Populus yunnanensis. Plant Cell Environ. 33, 1767-1778. doi: 10.1111/j.1365-3040.2010. 02182.x

Deinlein, U., Stephan, A. B., Horie, T., Luo, W., Xu, G., and Schroeder, J. I. (2014). Plant salt-tolerance mechanisms. Trends Plant Sci. 19, 371-379. doi: 10.1016/j.tplants.2014.02.001

Dudley, L. (2006). Ecological correlates of secondary sexual dimorphism in Salix glauca (Salicaceae). Am. J. Bot. 93, 1775-1783. doi: 10.3732/ajb.93.12.1775

Eckhart, V., and Chapin, F. (1997). Nutrient sensitivity of the cost of male function in gynodioecious Phacelia linearis (Hydrophyllaceae). Am. J. Bot. 84:1092. doi: $10.2307 / 2446152$

Garthwaite, A. J., von Bothmer, R., and Colmer, T. D. (2005). Salt tolerance in wild Hordeum species is associated with restricted entry of $\mathrm{Na}+$ and $\mathrm{Cl}$ - into the shoots. J. Exp. Bot. 56, 2365-2378. doi: 10.1093/jxb/eri229

Grabherr, M. G., Haas, B. J., Yassour, M., Levin, J. Z., Thompson, D. A., Amit, I., et al. (2011). Full-length transcriptome assembly from RNA-Seq data without a reference genome. Nat. Biotechnol. 29, 644-652. doi: 10.1038/nbt.1883

Greger, M., and Landberg, T. (1999). Use of Willow in Phytoextraction. Int. J. Phytoremediation 1, 115-123. doi: 10.1080/15226519908500010 the Fundamental Research Funds for the Central Universities of China (No. 2572020DY08), and the "Academic backbone" Project of Northeast Agricultural University of China (No. 18XG08).

\section{SUPPLEMENTARY MATERIAL}

The Supplementary Material for this article can be found online at: https://www.frontiersin.org/articles/10.3389/fpls.2020. 517962/full\#supplementary-material

Supplementary Figure 1 | Principal component analysis (PCA) based on gene expression from 24 sequenced samples.

Supplementary Figure 2 | Scatterplot of GO pathways enriched for differentially expressed genes (DEGs) in the roots of female and male plants of

S. linearistipularis. The rich factor is the ratio of the number of annotated DEGs in a given pathway term to the number of all genes annotated in the pathway term. The $Q$ value is the corrected $P$-value and ranges from 0 to 1 . The size of the circles indicates the number of genes. The top 20 enriched pathway terms in the KEGG database are listed. The red arrow indicates the significantly enriched response to the salt stress pathway.

Supplementary Figure 3 | Expression profiles of 108 salt-responsive DEGs in female and male roots under salt stress. The bar represents the scale of the expression levels of each DEGs ( $\log _{2}$ RPKM) in the heat map. The red rectangles represent the up-regulation of DEGs, and blue rectangles represent down-regulation. All information for each DEGs can be found in Supplementary Table S3.

Hughes, F. M. R., Johansson, M. E., Xiong, S. J., Carlborg, E., Hawkins, D., Svedmark, M., et al. (2010). The influence of hydrological regimes on sex ratios and spatial segregation of the sexes in two dioecious riparian shrub species in northern Sweden. Plant Ecol. 208, 77-92. doi: 10.1007/s11258-009-9689-x

Jiang, H., Peng, S., Zhang, S., Li, X., Korpelainen, H., and Li, C. (2012). Transcriptional profiling analysis in Populus yunnanensis provides insights into molecular mechanisms of sexual differences in salinity tolerance. J. Exp. Bot. 63, 3709-3726. doi: 10.1093/jxb/ers064

Jiang, Y., and Deyholos, M. K. (2009). Functional characterization of Arabidopsis $\mathrm{NaCl}$-inducible WRKY25 and WRKY33 transcription factors in abiotic stresses. Plant Mol. Biol. 69, 91-105. doi: 10.1007/s11103-008-9408-3

Karp, A., Hanley, S. J., Trybush, S. O., Macalpine, W., Pei, M., and Shield, I. (2011). Genetic improvement of willow for bioenergy and biofuels. J. Integr. Plant Biol. 53, 151-165. doi: 10.1111/j.1744-7909.2010.01015.x

Li, C., Xu, G., Zang, R., Korpelainen, H., and Berninger, F. (2007). Sex-related differences in leaf morphological and physiological responses in Hippophae rhamnoides along an altitudinal gradient. Tree Physiol. 27, 399-406. doi: 10. 1093/treephys/27.3.399

Li, L., Zhang, Y., Luo, J., Korpelainen, H., and Li, C. (2013). Sex-specific responses of Populus yunnanensis exposed to elevated CO2 and salinity. Physiol. Plant 147, 477-488. doi: 10.1111/j.1399-3054.2012.01676.x

Li, S. X., Liu, J. L., An, Y. R., Cao, Y. M., Liu, Y. S., Zhang, J., et al. (2019). MsPIP2;2, a novel aquaporin gene from Medicago sativa, confers salt tolerance in transgenic Arabidopsis. Environ. Exp. Bot. 165, 39-52. doi: 10.1016/j. envexpbot.2019.05.020

Li, Y., Duan, B., Chen, J., Korpelainen, H., Niinemets, U., and Li, C. (2016). Males exhibit competitive advantages over females of Populus deltoides under salinity stress. Tree Physiol. 36, 1573-1584. doi: 10.1093/treephys/tpw070

Liao, J., Song, H., Tang, D., and Zhang, S. (2019). Sexually differential tolerance to water deficiency of Salix paraplesia-A female-biased alpine willow. Ecol. Evol. 9, 8450-8464. doi: 10.1002/ece3.5175

Lou, D., Wang, H., and Yu, D. (2018). The sucrose non-fermenting-1-related protein kinases SAPK1 and SAPK2 function collaboratively as positive regulators of salt stress tolerance in rice. BMC Plant Biol. 18:203. doi: 10.1186/ s12870-018-1408-0 
Lv, S. L., Jiang, P., Tai, F., Wang, D. L. Y., Feng, J. J., Fan, P. X., et al. (2017). The V-ATPase subunit A is essential for salt tolerance through participating in vacuolar $\mathrm{Na}+$ compartmentalization in Salicornia europaea. Planta 246, 1177-1187. doi: 10.1007/s00425-017-2762-0

Matsuda, S., Nagasawa, H., Yamashiro, N., Yasuno, N., Watanabe, T., Kitazawa, H., et al. (2014). Rice RCN1/OsABCG5 mutation alters accumulation of essential and nonessential minerals and causes a high $\mathrm{Na} / \mathrm{K}$ ratio, resulting in a saltsensitive phenotype. Plant Sci. 224, 103-111. doi: 10.1016/j.plantsci.2014.04. 011

Maxwell, K., and Johnson, G. N. (2000). Chlorophyll fluorescence-a practical guide. J. Exp. Bot. 51, 659-668. doi: 10.1093/jxb/51.345.659

Melnikova, N. V., Borkhert, E. V., Snezhkina, A. V., Kudryavtseva, A. V., and Dmitriev, A. A. (2017). Sex-specific response to stress in Populus. Front. Plant Sci. 8:1827. doi: 10.3389/fpls.2017.01827

Mortazavi, A., Williams, B. A., McCue, K., Schaeffer, L., and Wold, B. (2008). Mapping and quantifying mammalian transcriptomes by RNA-Seq. Nat. Methods 5, 621-628. doi: 10.1038/nmeth.1226

Munns, R., and Tester, M. (2008). Mechanisms of salinity tolerance. Annu. Rev. Plant Biol. 59, 651-681. doi: 10.1146/annurev.arplant.59.032607.092911

Peng, S., Jiang, H., Zhang, S., Chen, L., Li, X., Korpelainen, H., et al. (2012). Transcriptional profiling reveals sexual differences of the leaf transcriptomes in response to drought stress in Populus yunnanensis. Tree Physiol. 32, 1541-1555. doi: 10.1093/treephys/tps110

Rahnama, A., James, R. A., Poustini, K., and Munns, R. (2010). Stomatal conductance as a screen for osmotic stress tolerance in durum wheat growing in saline soil. Funct. Plant Biol. 37, 255-263. doi: 10.1071/FP09148

Shi, H., Quintero, F. J., Pardo, J. M., and Zhu, J. K. (2002). The putative plasma membrane $\mathrm{Na}(+) / \mathrm{H}(+)$ antiporter SOS1 controls long-distance $\mathrm{Na}(+)$ transport in plants. Plant Cell 14, 465-477. doi: 10.1105/tpc.010371

Song, H., Cai, Z., Liao, J., Tang, D., and Zhang, S. (2019). Sexually differential gene expressions in poplar roots in response to nitrogen deficiency. Tree Physiol. 39, 1614-1629. doi: 10.1093/treephys/tpz057

Sun, J., Chen, S., Dai, S., Wang, R., Li, N., Shen, X., et al. (2009). NaCl-induced alternations of cellular and tissue ion fluxes in roots of salt-resistant and saltsensitive poplar species. Plant Physiol. 149, 1141-1153. doi: 10.1104/pp.108. 129494

Tian, F., Chang, E., Li, Y., Sun, P., Hu, J., and Zhang, J. (2017). Expression and integrated network analyses revealed functional divergence of NHX-type $\mathrm{Na}(+) / \mathrm{H}(+)$ exchanger genes in poplar. Sci. Rep. 7:2607. doi: 10.1038/s41598017-02894-8
Tozser, D., Magura, T., and Simon, E. (2017). Heavy metal uptake by plant parts of willow species: a meta-analysis. J. Hazard. Mater. 336, 101-109. doi: 10.1016/j. jhazmat.2017.03.068

Ueno, N., Suyama, Y., and Seiwa, K. (2007). What makes the sex ratio femalebiased in the dioecious tree Salix sachalinensis? J. Ecol. 95, 951-959. doi: 10.1111/j.1365-2745.2007.01269.x

Wang, W., Wang, Y., Du, Y., Zhao, Z., Zhu, X., Jiang, X., et al. (2014). Overexpression of Camellia sinensis $\mathrm{H} 1$ histone gene confers abiotic stress tolerance in transgenic tobacco. Plant Cell Rep. 33, 1829-1841. doi: 10.1007/ s00299-014-1660-1

Zhang, J., Yuan, H., Yang, Q., Li, M., Wang, Y., Li, Y., et al. (2017). The genetic architecture of growth traits in Salix matsudana under salt stress. Hortic. Res. 4:17024. doi: 10.1038/hortres.2017.24

Zhang, S., Jiang, H., Peng, S., Korpelainen, H., and Li, C. (2011). Sex-related differences in morphological, physiological, and ultrastructural responses of Populus cathayana to chilling. J. Exp. Bot. 62, 675-686. doi: 10.1093/jxb/ erq306

Zhou, A., Liu, E., Ma, H., Feng, S., Gong, S., and Wang, J. (2018). NaCl-induced expression of AtVHA-c5 gene in the roots plays a role in response of Arabidopsis to salt stress. Plant Cell Rep. 37, 443-452. doi: 10.1007/s00299-017-2241-x

Zhou, A., Ma, H., Liu, E., Jiang, T., Feng, S., Gong, S., et al. (2017). Transcriptome sequencing of Dianthus spiculifolius and analysis of the genes involved in responses to combined cold and drought stress. Int. J. Mol. Sci. 18:849. doi: 10.3390/ijms 18040849

Zhu, N., Cheng, S. F., Liu, X. Y., Du, H., Dai, M. Q., Zhou, D. X., et al. (2015). The R2R3-type MYB gene OsMYB91 has a function in coordinating plant growth and salt stress tolerance in rice. Plant Sci. 236, 146-156. doi: 10.1016/j.plantsci. 2015.03.023

Conflict of Interest: The authors declare that the research was conducted in the absence of any commercial or financial relationships that could be construed as a potential conflict of interest.

Copyright (C) 2020 Feng, Sun, Ma, Zhang, Ma, Qiao, Zhou, Bu and Liu. This is an open-access article distributed under the terms of the Creative Commons Attribution License (CC BY). The use, distribution or reproduction in other forums is permitted, provided the original author(s) and the copyright owner(s) are credited and that the original publication in this journal is cited, in accordance with accepted academic practice. No use, distribution or reproduction is permitted which does not comply with these terms. 